\title{
Femme et dame de courtoisie dans les manuscrits enluminés en France aux XIV ${ }^{\mathrm{e}}$ et $\mathrm{XV}^{\mathrm{e}}$ siècles
}

Thèse de doctorat en histoire de l'art médiéval, sous la direction de Daniel Russo, université de Bourgogne, et de Cécile Voyer, université de Poitiers, soutenue le 8 mars 2019

\section{Audrey Pennel}

\section{(2) OpenEdition}

\section{Journals}

\section{Édition électronique}

URL : https://journals.openedition.org/cem/16886

DOI : 10.4000/cem. 16886

ISSN : 1954-3093

Éditeur

Centre d'études médiévales Saint-Germain d'Auxerre

\section{Référence électronique}

Audrey Pennel, « Femme et dame de courtoisie dans les manuscrits enluminés en France aux xiv et $\mathrm{xv}^{\mathrm{e}}$ siècles », Bulletin du centre d'études médiévales d'Auxerre | BUCEMA [En ligne], 23.2 | 2019, mis en ligne le 31 janvier 2020, consulté le 22 septembre 2022. URL : http://journals.openedition.org/cem/ 16886 ; DOl : https://doi.org/10.4000/cem.16886

Ce document a été généré automatiquement le 22 septembre 2022.

\section{(c) (i) (3) (2)}

Creative Commons - Attribution - Pas d'Utilisation Commerciale - Partage dans les Mêmes Conditions 4.0 International - CC BY-NC-SA 4.0

https://creativecommons.org/licenses/by-nc-sa/4.0/ 


\section{Femme et dame de courtoisie dans les manuscrits enluminés en France aux XIV et $X^{\mathrm{e}}$ siècles}

Thèse de doctorat en histoire de l'art médiéval, sous la direction de Daniel Russo, université de Bourgogne, et de Cécile Voyer, université de Poitiers, soutenue le 8 mars 2019

\section{Audrey Pennel}

Femme et dame de courtoisie ne sont guère semblables, malgré la confusion visuelle que leur mise en image engendre parfois $^{1}$. Il est donc essentiel de comprendre quelles modulations et répétitions visuelles utilisent les artistes de la fin du Moyen Âge pour représenter ces deux figures féminines. L'image de la dame est-elle également représentative de la place de la femme dans la société du Moyen Âge tardif? En effet, la première est un personnage historique, rattaché à une réalité aristocratique. L'autre, née de

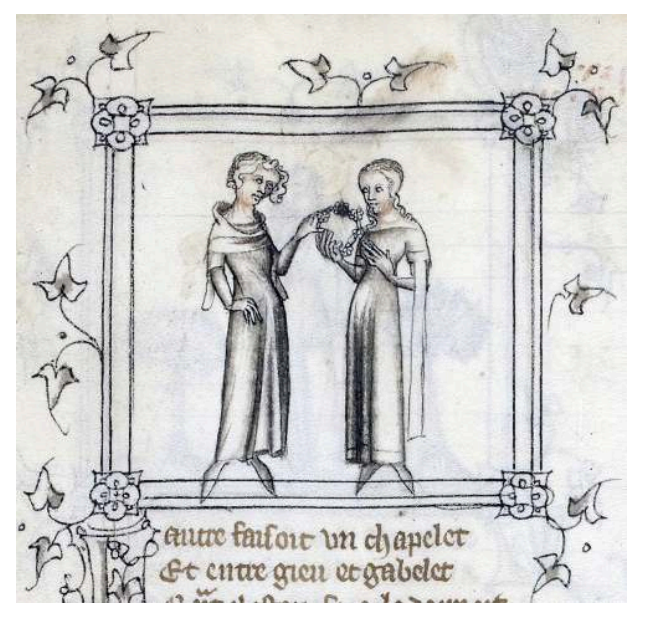
la lyrique, est une entité idéelle. Faut-il voir encore en ce Moyen Âge tardif un jeu, une mise en scène auxquels se livrent les femmes de la noblesse, les lettrées, durant des temps dédiés - jouent-elles à la Dame décrite dans les œuvres littéraires et poétiques ou le sont-elles, l'espace d'un instant, dans un contexte courtois -, ou bien la fin'amor conduit-elle progressivement, aux derniers siècles du Moyen Âge, à une modification du réel, à une transformation des rapports homme/femme et du statut des femmes ? Si le duo amant et dame appartient bien au même monde, celui formé par la dame et la femme permet de creuser un écart stimulant entre le réel et le fantasme, entre la réalité et la représentation. 
2 Cette thèse explore la place de la dame dans l'imaginaire et la société chevaleresque, l'approche "courtoise» des rites vassaliques et la question de l'identité du féminin désiré, entre allégorie, genre et transgression ${ }^{2}$. Notre principale problématique de travail examine la construction de la figure de « la dame » par et dans l'image, à travers les représentations des rituels dont elle est l'enjeu, la définition de son identité et son statut en tant qu'objet, agent ou sujet. Cette problématique repose, en partie, sur l'approche anthropologique de Maurice Godelier dans son ouvrage l'Idéel et le matériel' Cette approche est nécessaire pour appréhender l'étude des représentations de l'idéelle dame aimée s'inscrivant dans un imaginaire courtois, et celles de la femme noble, mise en scène dans ses réalités sociales.

3 Afin de mieux définir la place occupée par la femme et son " autre ", en tant que sujet ou objet, il est nécessaire de prendre en compte la signification des gestes, des positions et des attitudes des acteurs de la représentation.

\section{Cadre historiographique}

4 Le thème poétique d'un amour voué à une figure supérieure et inaccessible fait, depuis longtemps, l'objet d'études littéraires, sociales et historiques.

$5 \mathrm{Au}$ début de nos recherches, un grand nombre d'études concernant l'histoire des femmes au Moyen Âge et l'analyse littéraire du personnage de la dame nous est apparu. Nous nous sommes d'abord intéressés aux travaux de Georges Duby portant sur la femme médiévale face au regard de ses contemporains ${ }^{4}$. Les études de Sophie Cassagnes-Brouquet ont également retenu notre attention, notamment l'ouvrage Chevaleresses: une chevalerie au féminin ${ }^{5}$, qui dépeint les portraits de véritables femmes d'action à l'époque médiévale, jusqu'aux héroïnes littéraires. De même, les travaux de Didier Lett, dont Hommes et femmes au Moyen Âge, histoire du genre XII ${ }^{e}-\mathrm{XV}^{e}$ siècle $^{6}$, nous ont permis d'approfondir les rapports socioculturels entre féminin et masculin, à travers des questions de genre et d'identité.

6 En analysant le rôle des femmes à l'époque médiévale, nous avons considéré leur position face à l'exercice du pouvoir. Cette thématique est étudiée en 1988 par Mary Erler et Maryanne Kowaleski dans Women and Power in the Middle Ages ${ }^{7}$. Le rôle des femmes de pouvoir dans la production des arts et lettres à la fin du Moyen Âge, est, quant à lui, examiné dans une étude dirigée par Anne-Marie Legaré en 2007 : Livres et lectures de femmes en Europe entre Moyen Âge et Renaissance $e^{8}$, qui interroge les relations entre les femmes, l'écrit et les images.

$7 \mathrm{Au}$ commencement de notre thèse, les ouvrages entièrement consacrés à l'image des femmes au Moyen Âge tardif étaient moins nombreux. Ceux traitant spécifiquement des figurations du personnage de la dame étaient d'autant plus rares.

8 Concernant l'étude des représentations de rapports amoureux « courtois » à l'époque médiévale, nous nous sommes référés à The Medieval Art of Love de Michael Camille, publié en 1998, et à L'image de l'Amour Charnel au Moyen Âge de Florence Colin-Goguel, analysant des images issues de l'imaginaire ou révélatrices d'une réalité sociale codifiée. Nous nous sommes aussi intéressés à l'ouvrage "Plus est en vous", images de la femme au Moyen Âge (XIII ${ }^{e}-\mathrm{XV} V^{e}$ siècle) $)^{9}$. Cette étude, dirigée par Corinne Charles, repense l'imagerie de la femme et de la dame dans l'art, entre mythes, conventions sociales, morale théologique et modèles spirituels. Enfin, la thèse de Katherine Ilsley Sowley, $L a$ 
Tenture de la Dame à la licorne: la figure féminine au service de l'image masculine ${ }^{10}$, fut pertinente dans notre première approche, car consacrée à la figure de la dame à travers l'iconographie du cycle de cette tenture et aux réalités sociales entourant l'œuvre.

\section{Le corpus iconographique et méthodologie d'analyse}

Notre analyse cible à la fois le champ de l'histoire des femmes au Moyen Âge tardif, et l'évocation textuelle et visuelle de son « autre » idéelle, façonnée au cœur d'une société d'hommes. L'analyse des images de notre corpus est révélatrice de rapports entre la dame l'amant, où les liens entre sexe et genre deviennent parfois plus ambigus et où les rôles sont toujours renégociés.

Le nombre important de manuscrits enluminés du Roman de la Rose et des cycles peints du Tristan ou du Lancelot en prose, produits à cette période, explique la délimitation de notre corpus iconographique aux XIV et $\mathrm{XV}^{\mathrm{e}}$ siècles. C'est également le cas d'autres ouvrages poétiques et allégoriques - les œuvres de Guillaume de Machaut, de Christine de Pizan, le Champion des Dames ou le Livre des échecs amoureux-, fréquemment traités dans cette thèse. Le Moyen Âge tardif est propice au développement des thèmes courtois présents sur les calendriers enluminés des bréviaires et des livres d'Heures.

11 Notre travail repose sur l'étude de cent soixante-trois images, dont cent vingt-sept miniatures, issues de quatre-vingt-un manuscrits produits aux $\mathrm{XIV}^{\mathrm{e}}$ et $\mathrm{XV}^{\mathrm{e}}$ siècles, et de trente-six images de comparaisons. Les manuscrits étudiés ont été majoritairement produits pour des membres de la famille royale, des princes de Lys ou de grands seigneurs bibliophiles. Les femmes sont moins représentées que les hommes. Elles apparaissent néanmoins en tant que dédicataires - Isabeau de Bavière, Marie de Bourgogne, Valentine Visconti - ou commanditaires - par exemple, l'abbesse Marguerite de Bar commande le ms. 107 de la bibliothèque municipale de Verdun, pour son frère Renaud de Bar. Un volume de la Cité des Dames - LonDon, British Library, Harley 4431 -, écrit par Christine de Pizan, est aussi destiné à la reine Isabeau de Bavière.

12 L'analyse d'images se base sur une méthode sérielle proposée par Jérôme Baschet dans L'iconographie médiévale ${ }^{11}$, méthode mettant en réseau un important nombre d'images et permettant d'aborder plusieurs thématiques reposant sur un vaste corpus d'œuvres. L'insertion de trente-six images supplémentaires, prises en compte dans le cadre d'une analyse comparative, renforce l'approche sérielle. Ces œuvres, situées hors des frontières du corpus, sont analysées à titre ponctuel ou servent de références. Elles regroupent des supports variés - bois peint, sculpture, tapisserie ou objets précieux. De telles images, complémentaires, peuvent également provenir de manuscrits réalisés avant 1300 et après 1500, ou de volumes produits en dehors de la France (fig. 1). 
Fig. 1 - Répartition du pourcentage de manuscrits analysés par période

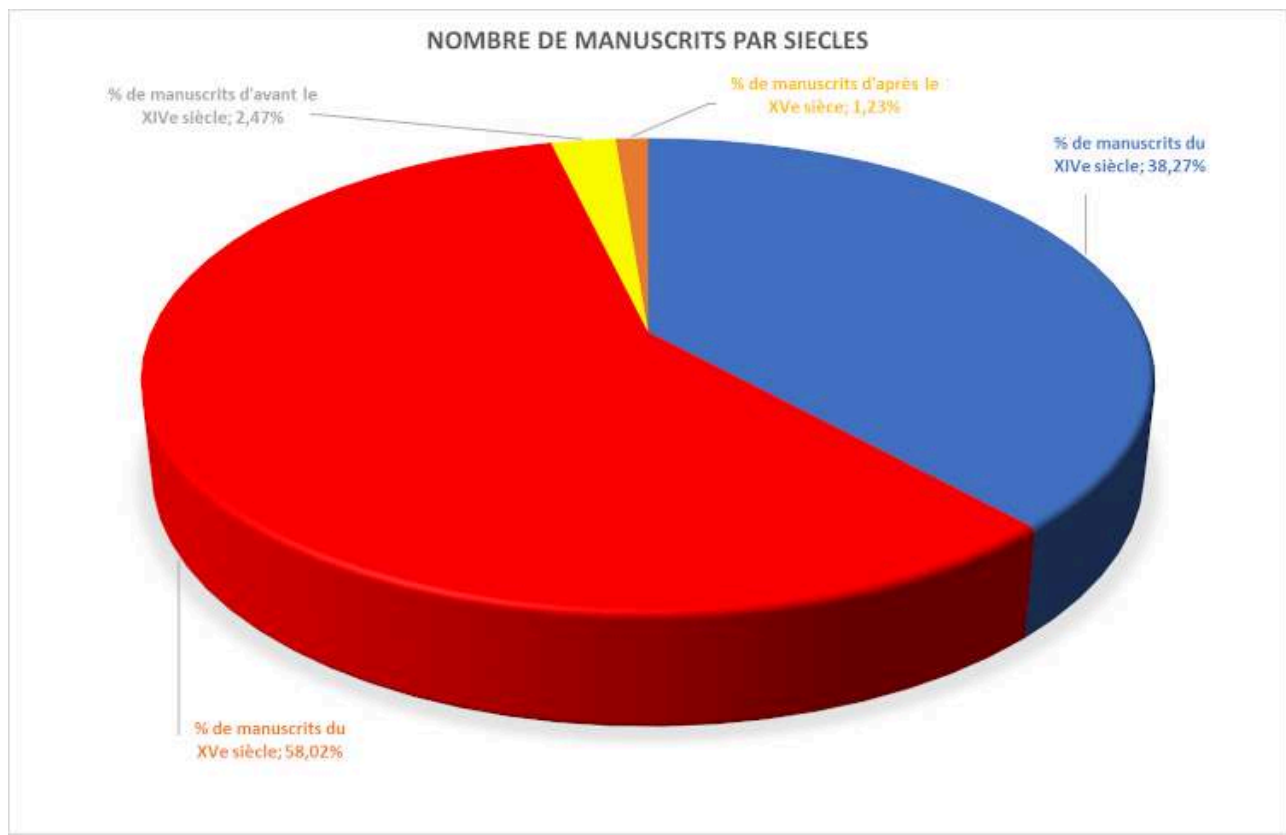

13 Afin d'approfondir correctement un champ iconographique déjà très riche dans la zone géographique ciblée, et d'éviter l'approche trop superficielle d'un corpus aux multiples thématiques, notre corpus iconographique est circonscrit aux frontières actuelles de la France $^{12}$.

14 La méthode va de pair avec une analyse des relations texte/image. Nous devions observer si la mise en scène des personnages et leurs attitudes au sein des représentations étaient fidèles à la description textuelle du récit original. Si oui, cela pouvait indiquer une bonne connaissance du texte de la part du miniaturiste ou du commanditaire. En revanche, nous avons également pu observer que l'image pouvait interpréter plus librement le texte ou en être entièrement détachée. Ainsi, le folio $53 \mathrm{du}$ manuscrit PARIS, BnF, fr. 580, contient une copie de l'Espitre à la Reine (fig. 2). 
Fig. 2 - Couple, Espitre à la Reine, Maître de l'Ovide, France (Paris), vers 1405

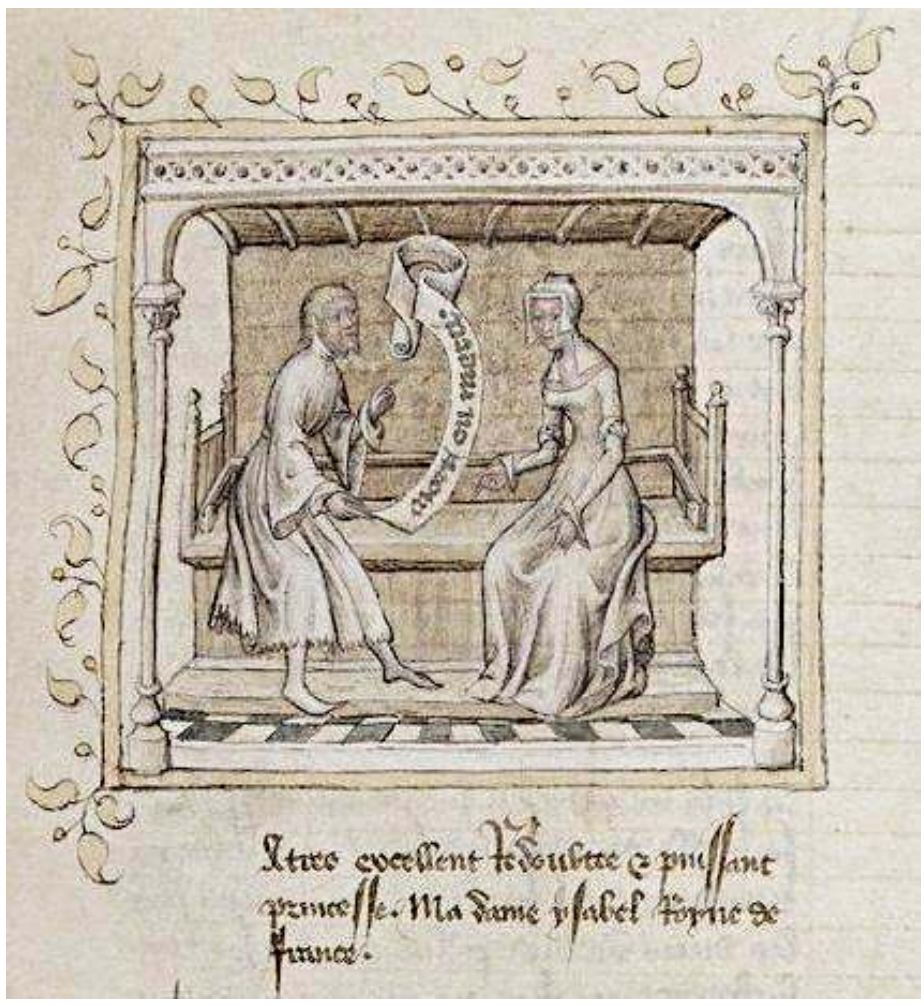

PARIS, BnF, fr. 580, fol. 53, cl. Gallica.

15 Une miniature précède la dédicace politique adressée par Christine de Pizan à la reine Isabeau de Bavière, en introduction à l'Épître: «Tres exxellent de grant haultesse / Couronnee poissant princesse, / Tres noble royne de France, / Le corps enclin vers vous m'adresce / En saluant par grant humblece. »

Un couple apparaît assis sur une banquette, dans un édicule ouvert reposant sur des colonnettes au premier plan. À gauche, l'homme présente un phylactère à la dame, tout en le désignant de sa main gauche. Les mots «mort ou merci » y sont inscrits. Le message n'entretient pas de lien avec le contenu textuel de l'épître. Cette demande de grâce semble davantage se substituer à certains gestes (la jonction des mains) ou à des attitudes (hommage), rappelant une supplique amoureuse. La dame arbore une robe et une coiffe simples. Elle ne dispose d'aucun attribut royal, ce qui l'éloigne de l'image d'Isabeau. Elle tend sa main droite, prête à recevoir les mots que lui transmet l'amant, par l'intermédiaire du phylactère. Le seul lien symbolique qu'entretiendrait l'image avec le contenu engagé de l'œuvre repose sur deux éléments : le motif échiqueté du sol, possible référence de la place occupée par la reine comme pièce maîtresse du jeu d'échecs politique, et le message « mort ou merci » contenu dans le phylactère, dont le caractère inhérent à la supplique amoureuse pourrait faire écho avec la demande de grâce que lance la poétesse à Isabeau de Bavière.

Afin de mieux noter les similitudes et les variations entre textuel et visuel, nous avons débuté nos analyses d'images par une introduction au texte choisi et, en son absence, au contexte entourant la représentation. Puis, nous avons commenté l'image, d'après une perspective sérielle, en comparant ce qui relevait du contenu scriptural et du commentaire visuel livré par le miniaturiste. 


\section{Les figures de la « dame »}

$$
\text { (fig. 3). }
$$

Fig. 3 - Répartition des images par thèmes dominants et croisements de séries

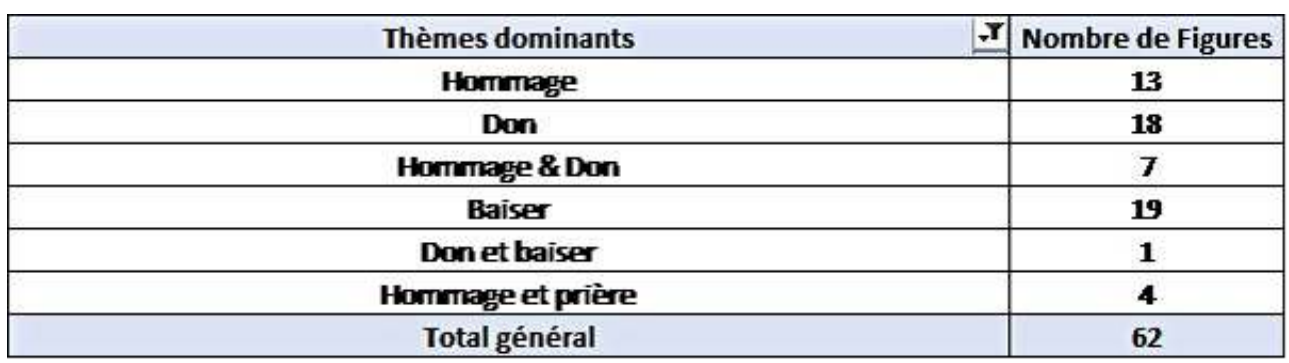

21 Trois principaux thèmes se distinguent également. Ils concernent soixante-deux images analysées sur cent vingt-sept. Il s'agit de l'hommage, du don et du baiser. Ces thématiques peuvent aussi s'entrecroiser à l'intérieur de certaines œuvres visuelles. L'hommage est le plus éminent du champ sériel, avec vingt-quatre occurrences au total. Il s'insère au cœur d'une importante réflexion consacrée aux relations entre imaginaire courtois et réel social. Le troisième chapitre de la thèse y est d'ailleurs consacré.

Il est suivi par le thème du don regroupant dix-huit images, auxquelles on inclut sept autres également associées à l'hommage, et une du baiser. Enfin, la thématique du baiser contient au total vingt images, dont une est associée au don.

Les gestes et les postures relevant de l'hommage amoureux fonctionnent comme une réinterprétation de la cérémonie témoignant de l'allégeance d'un vassal à son seigneur. L'hommage est un acte principalement masculin dans notre corpus. L'amant est souvent représenté à genou, les mains jointes ou tenant la main de sa dame, en posture d'humilité (fig. 4 et 5). L'hommage marque le début d'un service d'amour entre l'impétrant et sa dame souveraine. 
Fig. 4 - Homme agenouillé devant une dame, Bestiaire d'Amour, France (Metz), premier quart du XIV siècle

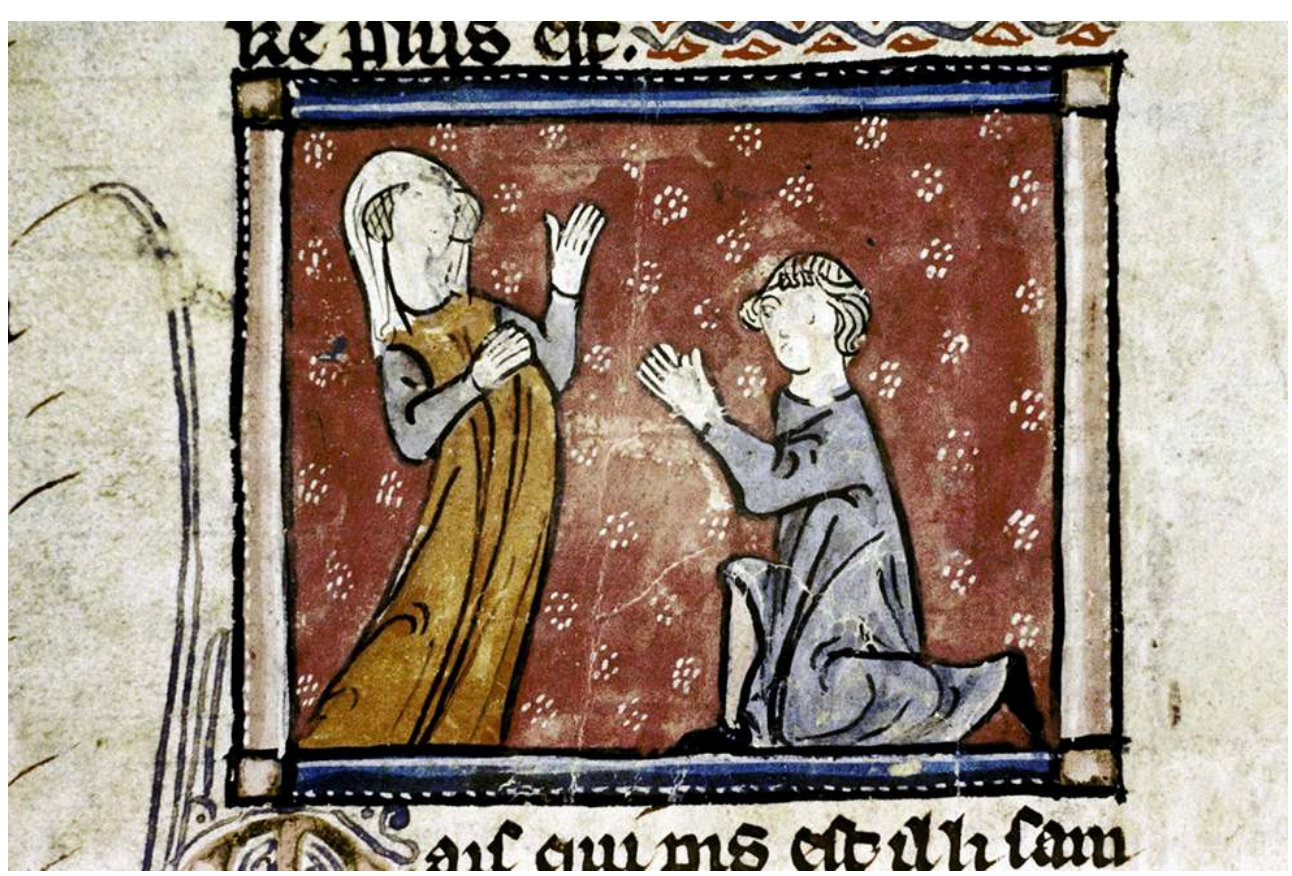

oxfoRd, Bodleian Library, Douce 308, fol. 100, cl. Bodleian Libraries, University of Oxford.

Fig. 5 - Machaut et sa dame, Le Remède de Fortune, Maître du Remède de Fortune, France (Paris), $1350-1356$

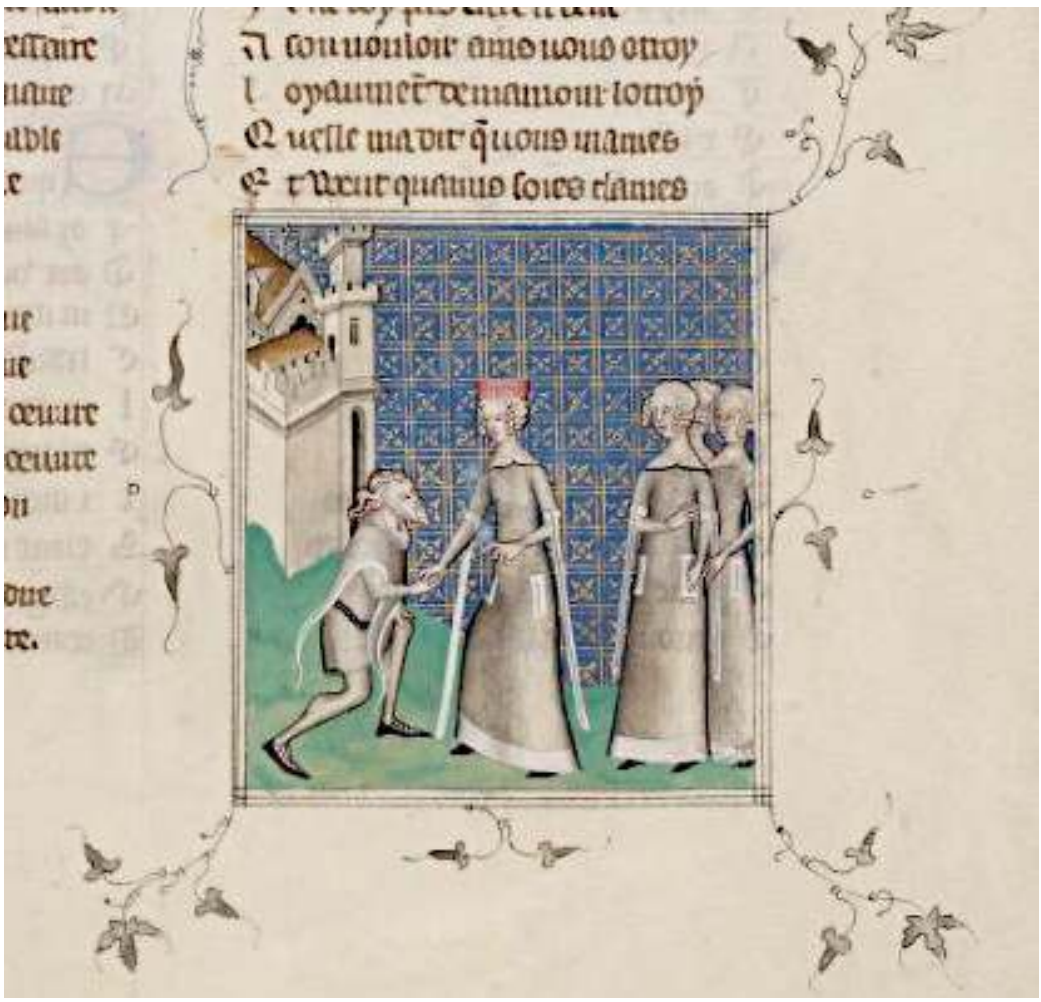

PARIS, BnF, fr. 1586, fol. 54, cl. Gallica. 
Le baiser amoureux est la deuxième étape qui scelle une promesse de fidélité entre la dame et son bien-aimé. Au récurrent geste d'enlacement accompagnant le baiser sur la bouche (fig. 6), s'ajoutent alors des variantes visuelles, telles la main sur la joue significative d'amour ${ }^{13}$ (fig. 7), ou la main sur la poitrine évoquant une volonté de possession (fig. 8$)^{14}$.

Fig. 6 - Coronis et son amant, Livre du Voir-Dit, Perrin Remiet, France (Paris), vers 1390

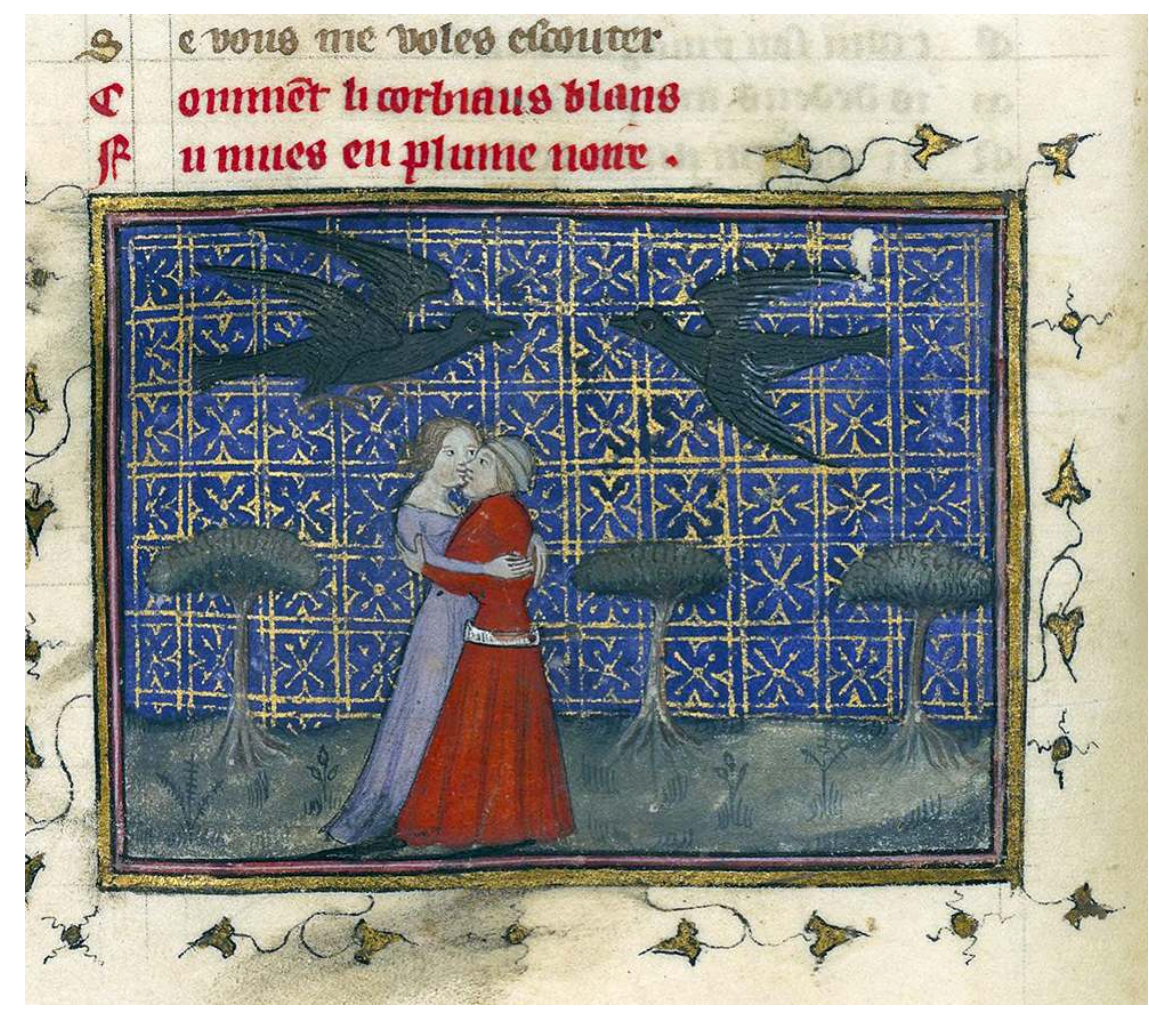

PARIS, BnF, fr. 22545, fol. 189v, cl. Gallica. 
Fig. 7 - Baiser de Lancelot et Guenièvre, Lancelot en prose, Évrard d'Espinques, France (Ahun), vers 1470

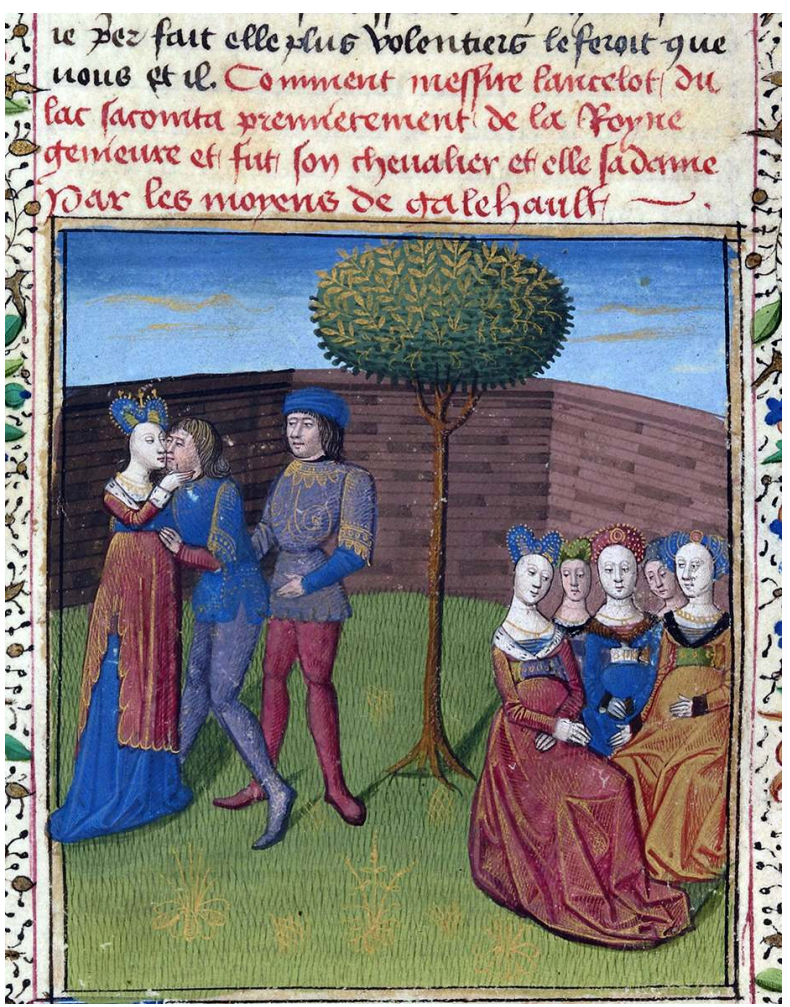

PARIS, BnF, fr. 112 (1), fol. 101, cl. Gallica.

Fig. 8 - Robin donnant un baiser à Marion devant Gauthier, Jeu de Robin et de Marion, France (Paris), premier quart du XIve siècle

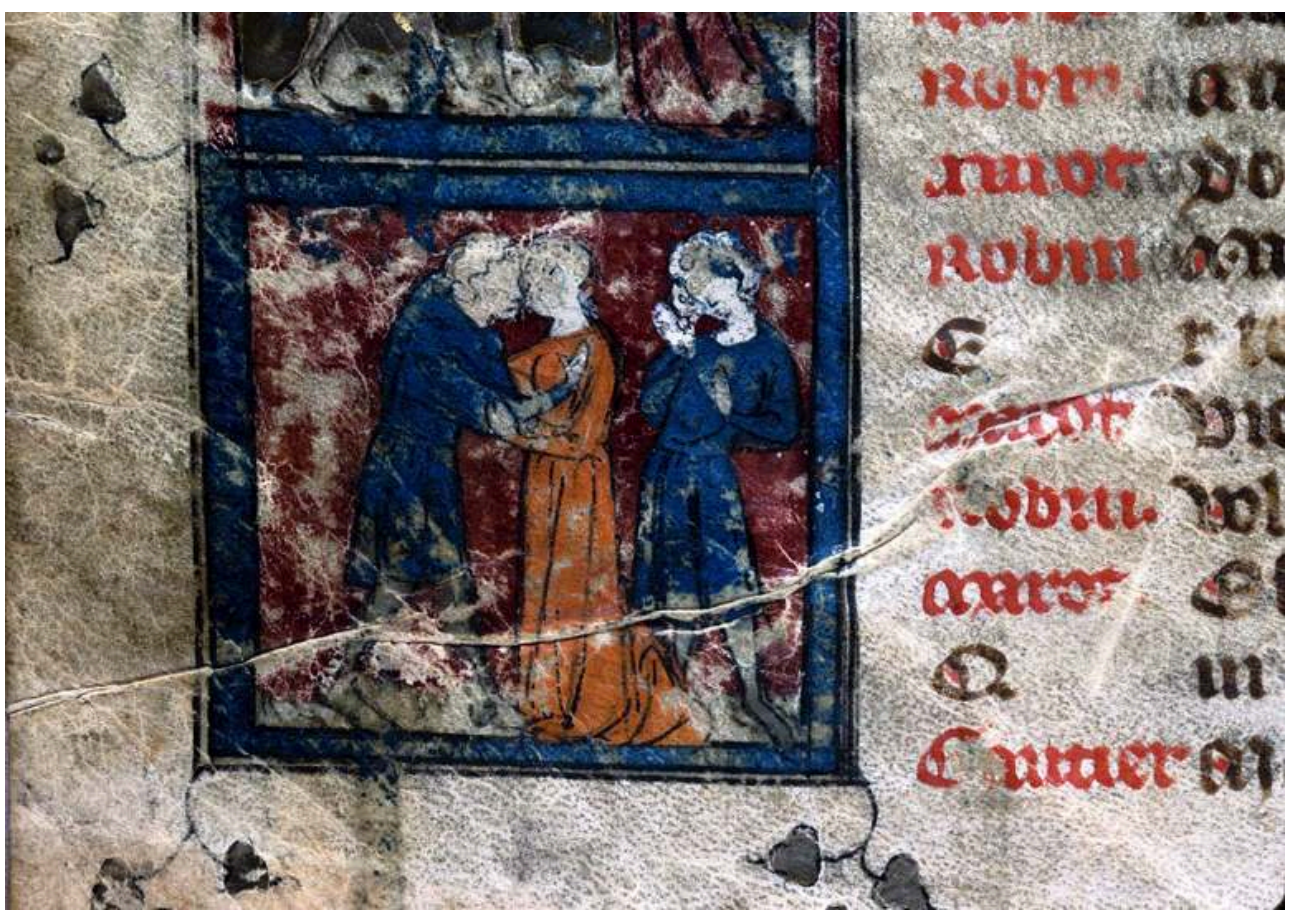

AIX-EN-Provence, Bibliothèque municipale, ms. 166, fol. 6v, cl. IRHT-CNRS 
Le commencement d'une relation amoureuse est également concrétisé par le don de présents aussi variés que peuvent l'être le poème, les anneaux, le chapel fleuri (fig. 9).

Fig. 9 - Une amante déloyale offre un chapel de fleurs à son ami, Dit du Lion, France (Reims), vers $1372-1377$

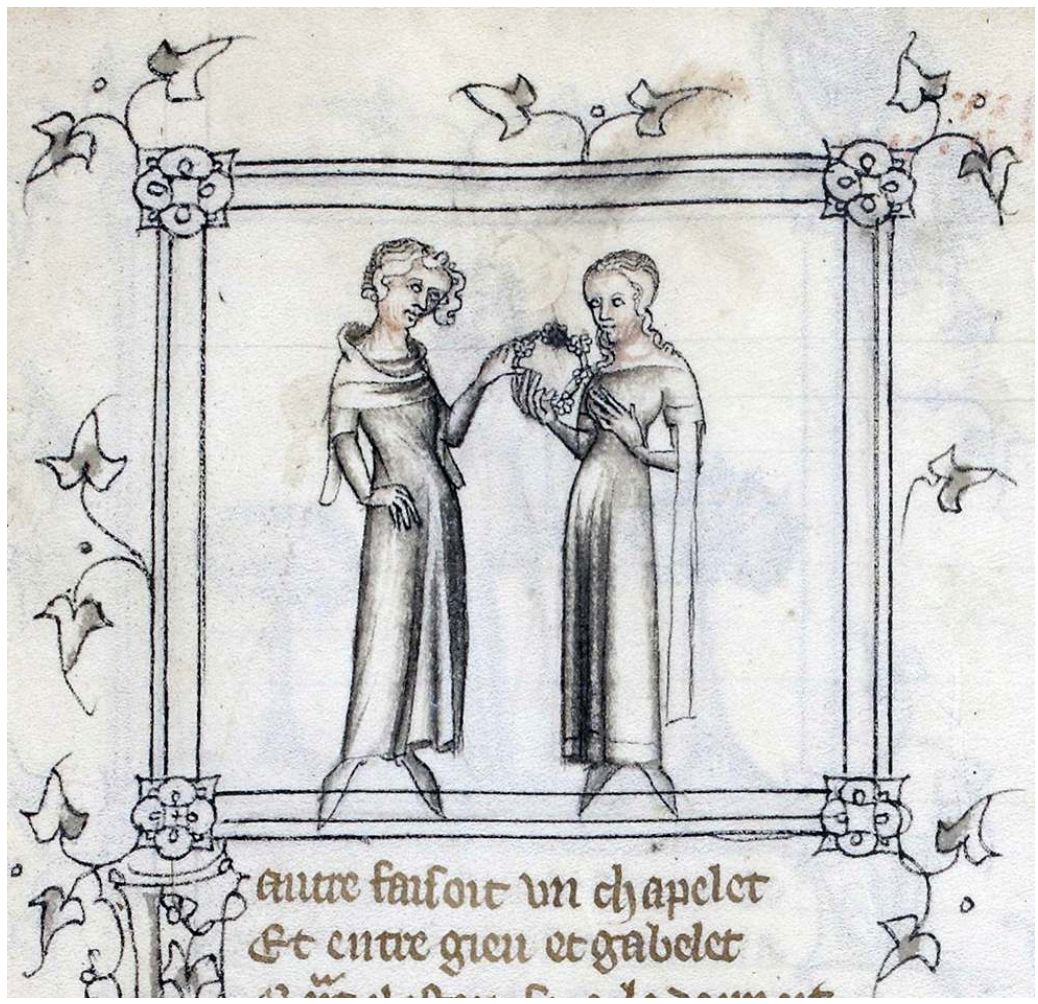

PARIS, BnF, fr. 1584, fol. 92v, cl. Gallica.

Le don relève des usages sociaux aristocratiques parfois liés à la mise en scène du pouvoir de la femme noble, comme à travers les miniatures des dédicaces (fig. 10). 
Fig. 10 - Philippe Bouton présentant son livre à Marie de Bourgogne, Le Mirouer des Dames, France (Bourgogne), vers 1480

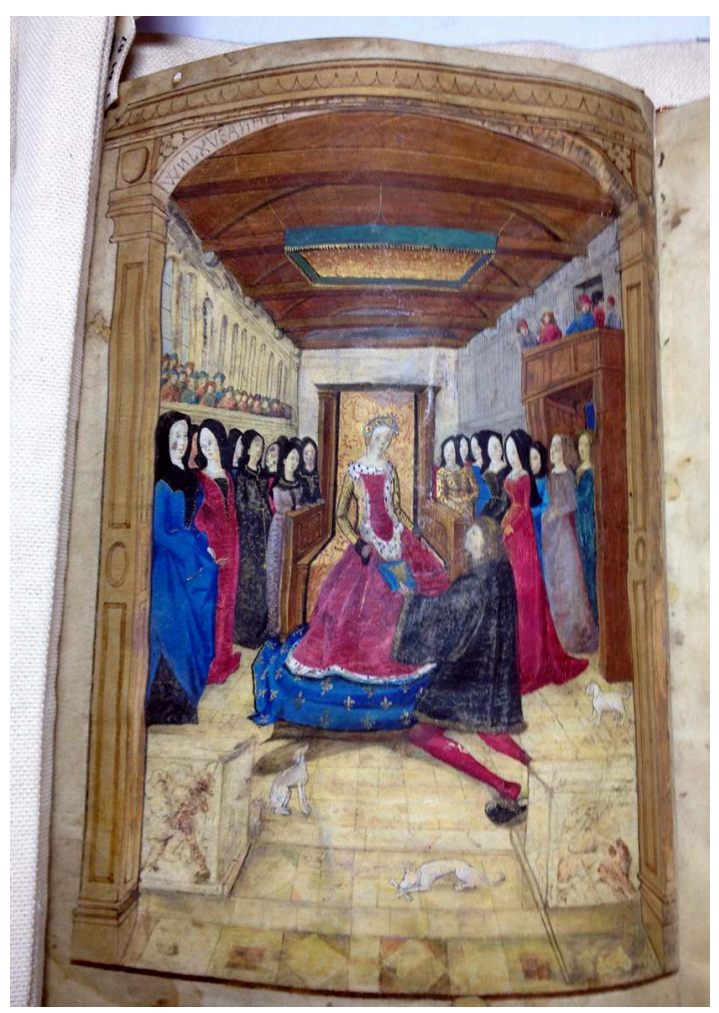

DIJon, Bibliothèque municipale, ms. 3463, fol. 1, cl. A. Pennel (2014)

De même, ce don peut être de nature métaphorique ou impliquant un don de soi (don du cœur ou épreuves) qui rappelle l'abnégation éprouvée par l'amant pour sa dame.

\section{L'identité féminine}

La question de l'identité féminine repose ici autant sur le statut objet/sujet des personnages que sur leur reconnaissance genrée. Parfois mise en scène comme une dame, la femme noble peut être identifiable socialement grâce à la lecture de ses armoiries et de ses emblèmes reproduits à l'intérieur de l'image (fig. 11). 
Fig. 11 - Honoré Bouvet offrant son ouvrage à Valentine Visconti, Apparition Maistre Jean de Meun

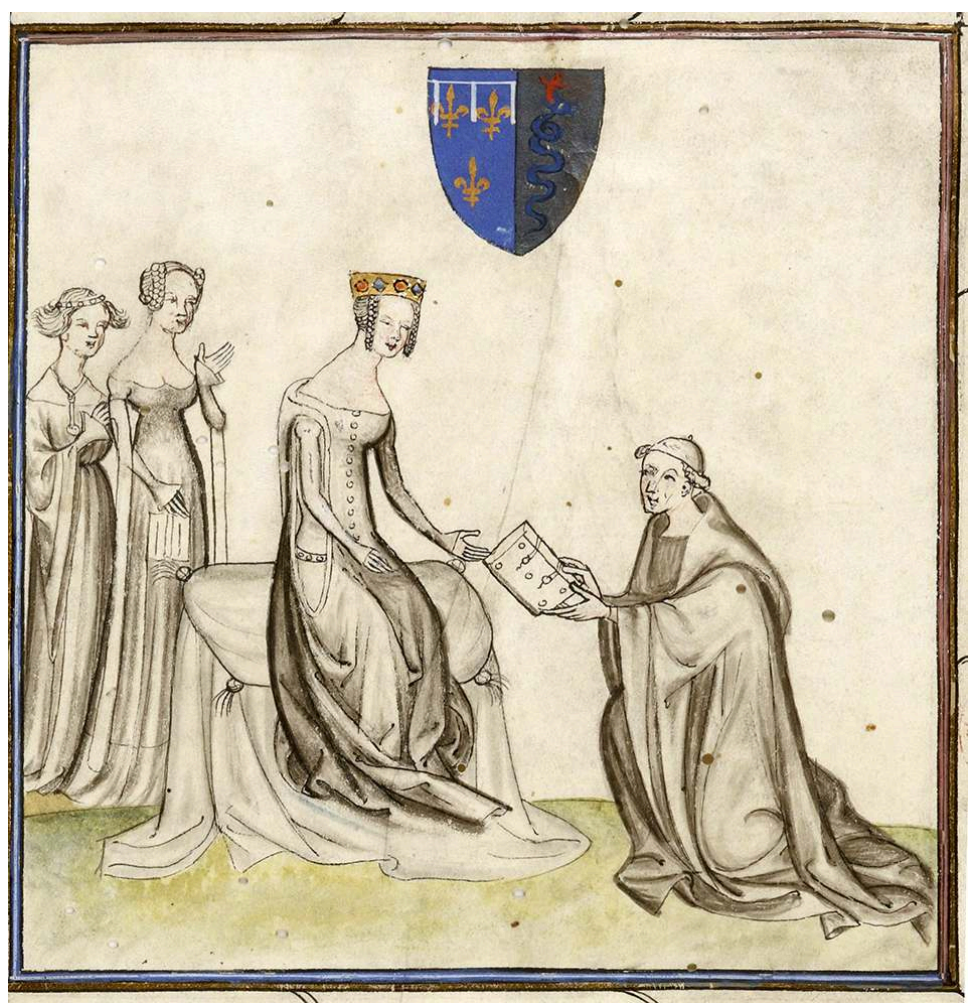

PARIS, BnF, fr. 811, fol. 1v, cl. Gallica.

Ainsi, sur une miniature montrant une scène de dédicace à Valentine Visconti, les armoiries de la duchesse d'Orléans «mi-partie Orléans aux lis, mi-partie Visconti à la guivre d'azur engoulant un enfant de gueules » apparaissent dans la partie supérieure de l'image. La présence de tels emblèmes permet également de légitimer le pouvoir du sujet féminin représenté. En l'absence d'armoiries, par exemple, c'est une autre codification visuelle qui prend le relai pour montrer le statut social. Dans le cas de la miniature de dédicace adressée à Marie de Bourgogne, il s'agit de certains motifs, comme le tapis fleurdelisé, ainsi que deux bas-reliefs au premier plan, où figurent le combat d'Hercule contre le lion de Némée et la victoire de Samson sur le lion (cf. fig. 10). Or, il se trouve que le grand-père et le père de la duchesse tenaient Hercule en haute estime et considérait les prouesses de Samson comme la préfiguration du salut de l'humanité, concrétisé par la venue du Christ.

Les représentations galantes de la duchesse Marie de Berry, de la reine Jeanne $\mathrm{I}^{\mathrm{re}}$ de Naples ou de la princesse Louise de Savoie témoignent également d'une volonté de mise en scène politique ou de démonstration d'un mode de vie curial esthétisé, comme dans certaines miniatures des Très riches Heures du duc Jean de Berry (fig. 12). 
Fig. 12 - Chevauchée aristocratique, scène des fiançailles, mois de mai, Les très riches Heures du duc de Berry

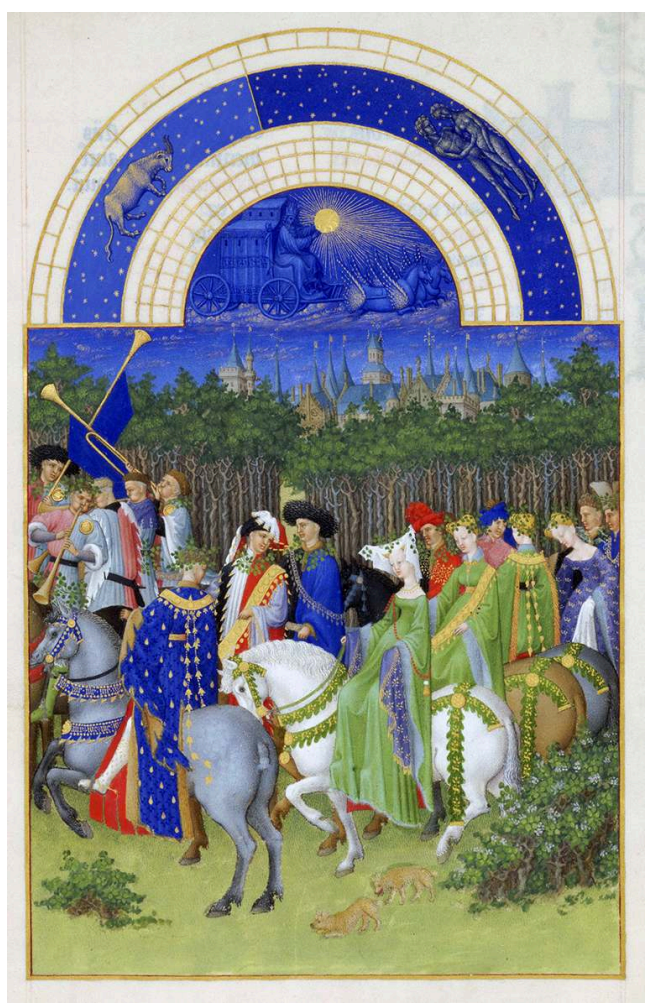

chantilly, Musée Condé, ms. 65, fol. 5, cl. Wikimedia Commons.

Les identités de la dame font, de même, l'objet d'une étude particulière au sein de notre troisième partie, entre transformations allégoriques et réification. Certains objets utilisés dans les scènes de loisirs amoureux sont caractéristiques d'un féminin métaphorique, tels la harpe et le luth, associés aux sentiments et au corps de la dame (fig. 13), ou le jeu d'échecs, métaphore pour le corps de la dame à conquérir. 
Fig. 13 - Concert amoureux, Le Champion des Dames, Maître du Dit de Remord et de Cheminant, France (Lille), 1475

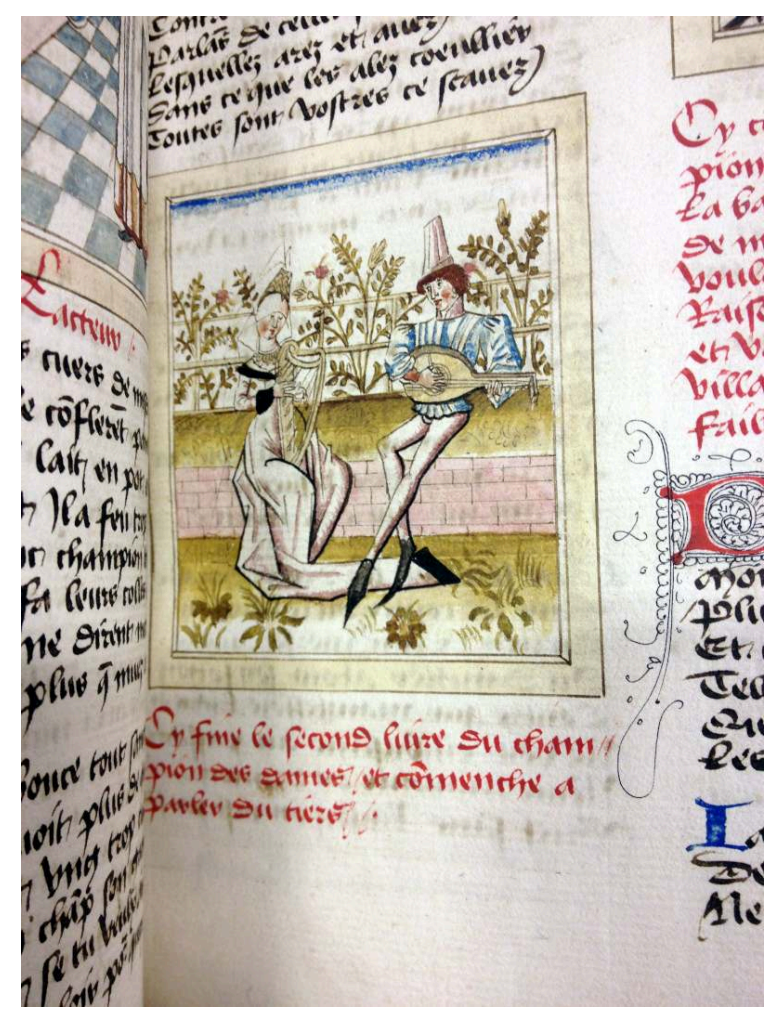

Paris, BnF, fr. 841, fol. 79, cl. A. Pennel (2015)

Le corps féminin désiré peut être, de plus, réifié à travers les thèmes visuels du château d'amour assiégé, du jardin fortifié ou encore de la rose au buisson épineux (fig. 14). 
Fig. 14 - L'amant embrassant la rose, Le Roman de la Rose, France (Paris), vers 1350

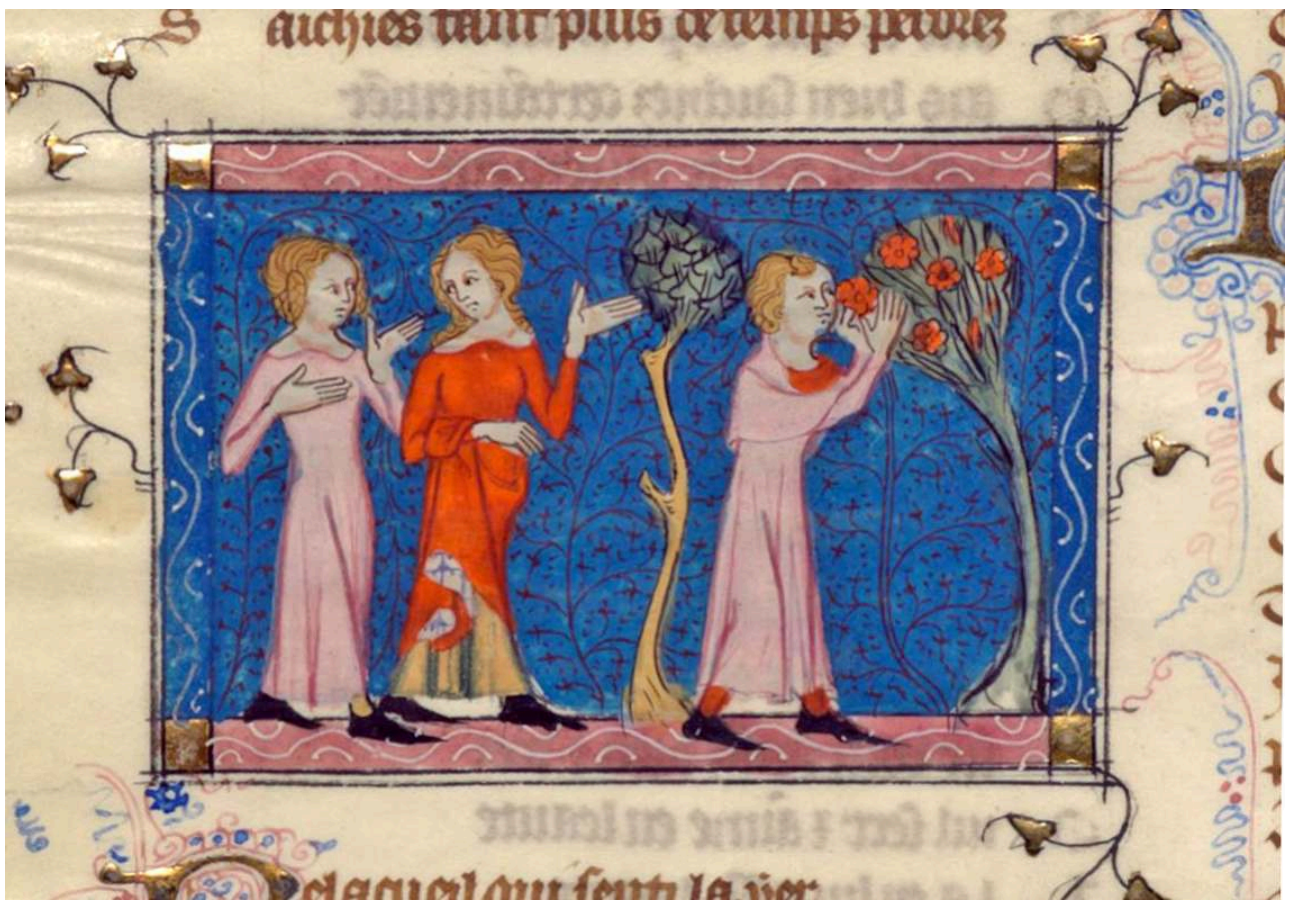

PARIS, BnF, fr. 1567, fol. 26v, cl. Gallica

Une certaine confusion des genres émane également de la nudité des corps féminins et masculins, mis en scène conjointement et, parfois, peu différenciés dans leurs attributs sexuels: c'est ainsi le cas d'autres personnifications de genre ambigu, comme la personnification de Bel Accueil dans le Roman de la Rose. L'identité de ce personnage, masculin, dans le texte, se mêle à celle de la dame aimée, y compris dans ses représentations. Sous le pinceau du miniaturiste, le personnage devient ainsi la dame elle-même, s'abandonnant entre les bras de l'amant (fig. 15) ou menant ce dernier jusqu'au buisson de roses, avec la complicité de Vénus.

Fig. 15 - Bel Accueil en discussion avec la Vieille, puis embrassant un amant, Le Roman de la Rose, France (Paris), deuxième quart du XIV siècle

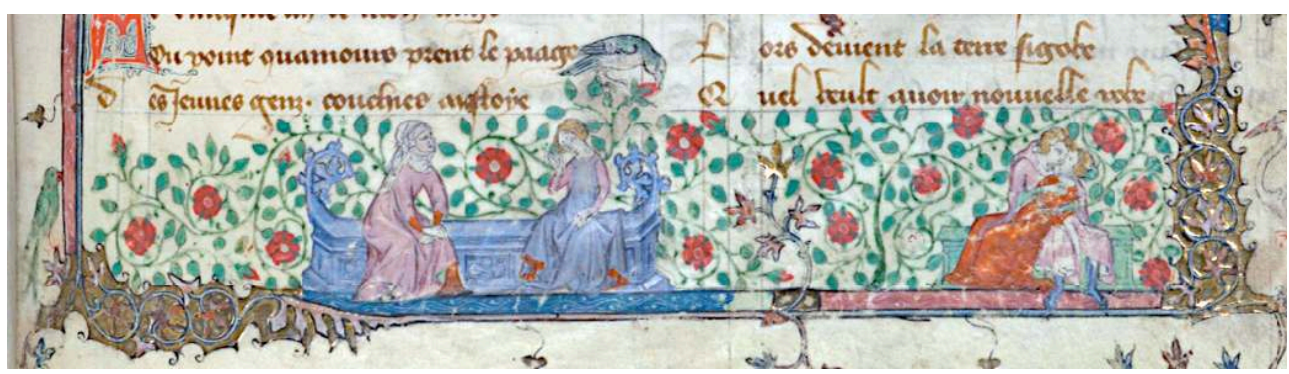

PARIS, BnF, fr. 1572, fol. 3, cl. Gallica.

\section{Principaux apports et prolongements}

Au terme de la thèse, nous avons relevé trois principaux apports. 

d'images s'insèrent à l'intérieur d'un large champ problématique, à savoir littéraire, historique, sociologique, iconographique et anthropologique, ou encore orienté vers la question du genre. Nous avons pu interroger la survivance de certains codes et modèles de représentation pour la dame idéale, mais aussi interroger, plus précisément, l'évolution (ou l'involution) du statut des femmes à la fin du Moyen Âge. L'interdisciplinarité permet d'éclairer le rôle de la femme médiévale au cœur des rites sociaux du pouvoir nobiliaire comme du mécénat artistique. Nous appréhendons alors la manière dont ce rôle est réinterprété, modifié et métamorphosé au sein des représentations courtoises.

Ensuite, la méthode sérielle nous a permis de dégager un hyperthème - la dévotion à la dame - et de révéler les thèmes dominants de l'hommage, du don et du baiser, tout en les inscrivant à l'intérieur des deux axes majeurs, ceux des rites sociaux et de l'identité. L'approche sérielle est un choix méthodologique avisé pour traiter un dense corpus iconographique et pour envisager, puis rassembler, la multiplicité des thèmes relatifs à la femme noble vénérée et à l'idéelle dame aimée, jusque-là absents du champ des études en histoire de l'art médiéval.

Enfin, les bases de données extraites du corpus des manuscrits synthétisent des informations sur les commanditaires, les artistes mais aussi les lieux et les dates de production entourant la réalisation des volumes enluminés. Ces informations seront réutilisables pour de prochaines études ou pourront être enrichies par tout chercheur travaillant sur les manuscrits des $\mathrm{XIV}^{\mathrm{e}}$ et $\mathrm{XV}$ e siècles.

Notre thèse a fait émerger, ainsi, des réflexions plurielles qui pourraient faire l'objet d'études approfondies.

Le Livre des Cent Ballades de Jean Le Sénéchal met en scène le personnage féminin, «Guignarde », une dame conseillant au jeune amant d'aimer en cent lieux, à l'opposé des leçons de loyauté d'un vieux chevalier. Les concepteurs du ms. $491^{15}$, une copie de l'œuvre conservée à la bibliothèque du musée Condé à Chantilly, évoluant dans l'entourage de René d'Anjou, renomment «Guignarde » Maquerelle et produisent une figure à deux identités, qu'il paraît intéressant d'étudier, entre séduction et personnification d'un vice.

De même, notre analyse du jeu cynégétique basé sur des manuscrits des XIV et $\mathrm{xV}^{\mathrm{e}}$ siècles, nous a permis de constituer une série d'images sur le thème de la chasse à la licorne, en particulier autour de la figure de la jeune vierge protectrice de l'animal.

41 Il nous semble également intéressant d'aborder plus avant le cas de certaines personnifications allégoriques (tel Bel Accueil) ou mythologiques, à la croisée des genres, entre Moyen Âge et Renaissance. aussi faire l'objet d'une étude comparative entre les miniatures médiévales et les peintures du XVI ${ }^{\mathrm{e}}$ siècle.

Enfin, les codes visuels prônant la dame en tant qu'archétype de désir au Moyen Âge questionnent notre rapport à l'image de la femme aujourd'hui. Certains canons esthétiques diffèrent des réalités matérielles entourant les femmes, mais parviennent à établir de nouvelles normes au sein de la société. Cette analyse, à la croisée de l'histoire, de la sociologie et de l'histoire de l'art, apporterait une nouvelle approche quant à l'évolution des modèles de perfections alors incarnés par un féminin idéel. 
Reçu : 31 juillet 2019 - Accepté : 6 novembre 2019

\section{NOTES}

1. Nous choisissons de distinguer, dans notre travail de thèse, la femme dans son genre ainsi que son environnement social, de la Dame comme représentation idéelle issue de la lyrique courtoise et parfois si proche du féminin aristocratique mis en scène. Femme et dame sont, en premier lieu, envisagées comme deux entités, l'une réelle, l'autre idéelle, dont l'identité et les rôles varient selon les thématiques étudiées. Leur mention doit donc être laissée au singulier.

2. Cette thèse a été soutenue devant un jury composé de Didier Lett (président et rapporteur), d'Anne-Marie Legaré (rapporteur), de Bruno Boerner, de Jean-Marie Fritz, de Daniel Russo (directeur de thèse) et de Cécile Voyer (codirectrice de thèse).

3. M. GODELIER, L'idéel et le matériel. Pensée, économies et sociétés, Paris, 2010.

4. Notamment Mâle Moyen Âge. De l'amour et autres essais, Paris, 1988, Femmes, mariages-lignages, $\mathrm{XII}^{e}$-XIVe siècles, Bruxelles, 1992, ou en codirection avec Michelle Perrot, Histoire des femmes en Occident, t. 2, Paris, 2002.

5. S. CASSAGnes-Brouquet, Chevaleresses. Une chevalerie au féminin, Paris, 2013.

6. D. LETT, Hommes et femmes au Moyen Âge. Histoire du genre, XII ${ }^{e}-X V^{e}$ siècle, Paris, 2013.

7. M. ERLER et M. KOWALESKI, Women and Power in the Middle Ages, Athens, University of Georgia Press, 1988.

8. A.-M. LegARÉ (dir.), Livres et lectures de femmes en Europe entre Moyen Âge et Renaissance, Turnhout, 2007.

9. C. Charles (dir.), "Plus est en vous »: images de la femme au Moyen-Âge (XIII ${ }^{e}-X V^{e}$ siècle), catalogue d'exposition, Bilbao, 2011.

10. K. ILSLEy SOWley, La Tenture de la Dame à la licorne: la figure féminine au service de l'image masculine, thèse de doctorat en histoire de l'art, université de Strasbourg, 2012.

11. J. BASCHET, L'iconographie médiévale, Paris, 2008.

12. Étudier les manuscrits enluminés à partir des frontières actuelles de la France nous a permis d'avoir une vision claire des productions dans un territoire donné, de faciliter les analyses codicologiques ainsi que l'introduction de nos résultats d'étude dans les calculs de notre enquête d'espace. Ce cadre, certes anachronique vis-à-vis de la période médiévale, est également commode pour circonscrire le corpus.

13. F. GARNIER, Le langage de l'image au Moyen Âge, t. 2 (Grammaire des gestes), Paris, 1989, p. $120-121$.

14. Selon Chiara Frugoni, si poser une main sur la poitrine d'un homme signifie qu'on le fait prisonnier, une main posée sur la poitrine d'une femme indique le pouvoir que détient le mari sur son épouse : C. FRUGONI, Le Moyen Âge par ses images, Paris, 2015, p. 35-37. Ce geste présent sur la miniature d'un volume du Jeu de Robin et Marion (AIX-EN-PROVENCE, Bibliothèque municipale, ms. 166, fol.6v) révélerait donc la volonté de possession de Robin, celui-ci ne souhaitant que personne, ni le chevalier, ni même Gauthier figuré ici, ne vienne lui prendre Marion.

15. Ils ne sont pas connus ou désignés en tant que "Maîtres ", néanmoins Gaston Raynaud signale leur appartenance à l'entourage de René d'Anjou : G. RAYNAUD, Les Cent Ballades : poème du XIVe siècle composé par Jean le Seneschal; avec la collaboration de Philippe d'Artois, comte d'Eu, de Boucicaut le jeune et de Jean de Crésecque, Paris, 1905, p. 169. 
INDEX

Mots-clés : amour courtois, fin'amor, manuscrit, enluminure, iconographie, dame, amant, France, histoire sociale, Moyen Âge tardif, rites, aristocratie, imaginaire, hommage, identité, don, baiser, amour charnel, transgression, personnification, allégorie, genre, codification, sérialité

\section{AUTEUR}

AUDREY PENNEL

Université de Bourgogne 